\title{
Some Problems of Ionospheric Nonlinearities
}

\author{
Donald H. Menzel ${ }^{1}$
}

(Received February 20, 1964; revised August 5, 1964)

\begin{abstract}
The theoretical solution of the problem of the propagation of radio waves through an ionized medium depends on Maxwell's well-known equations, together with the equation of motion of a free electron. This last equation, in general, contains a large number of terms. In addition to the purely electromagnetic terms, there are others expressing the interaction of the electron with the medium itself. The solution requires one to determine the complex index of refraction as a function of the applied radio frequency. The problem is even more involved, in some cases, because magnetohydrodynamical terms may enter into the expression. Sound waves or shock waves in the medium, for example, can impress themselves on the complex index of refraction. As a result, dynamical and magnetohydrodynamical interactions can lead to the coupling of fields that otherwise would be independent.
\end{abstract}

\section{Introduction}

I wish, first of all, to extend a welcome on behalf of Dr. Ernest K. Smith and myself, cochairmen of this conference. My task this morning would have been much lighter if only two distinguished Soviet scientists, Prof. V. L. Ginsburg and Prof. A. V. Gurevich, had been able to accept our invitation. Their joint paper [1960] on "Nonlinear Phenomena in a Plasma Located in an Alternating Electromagnetic Field" is a classic. It reviews and extends the basic principles and applies them to an examination of general nonlinear problems.

Their paper is far too long and detailed for me to review here. Perhaps I should just commend it to your attention and then sit down. But such brevity is hardly in the tradition of conference chairmen. Besides, I should like to discuss some relatively unfamiliar nonlinear processes that may occur in certain types of ionized plasmas.

Various contributors to this conference have asked me to explain the qualifying adjective, "nonlinear," which defines the type of processes we are discussing. here today. Let me say, first of all, that "nonlinear" does not signify "irregular" or "nonuniform." The mathematician, the physicist, and the engineer each has his own definition of "nonlinear." At first sight these explanations may seem to be very different, but they are actually only alternate ways of expressing the same basic facts.

To the mathematician, quantities such as $E, \frac{d E}{d t}$, $\frac{d^{2} E}{d t^{2}}$, etc., are linear in that they depend only on the first power of $E$. Differential equations involving these quantities have solutions of the form:

$$
E=f(t) .
$$

\footnotetext{
${ }^{1}$ Harvard College Observatory, Cambridge, Mass.
}

And if two solutions are simultaneously present, the complete solution is simply the sum of the two, or

$$
E=f_{1}(t)+f_{2}(t) .
$$

Mathematicians call this the principle of superposition.

Mathematical quantities involving the square, or higher power, or cross product of the foregoing, such as $E^{2},\left(\frac{d E}{d t}\right)^{2}, E \frac{d E}{d t}$, etc., are nonlinear. Mathematically, we recognize the nonlinearity because the complete solution is not the sum of two elementary solutions. Cross-product terms exist. The two disturbances react on one another. For example,

and so on.

$$
E^{2}=f_{1}^{2}(t)+2 f_{1}(t) f_{2}^{2}(t)+f_{2}(t),
$$

Physically, E may represent an electric field that varies with the time. Any number of electromagnetic fields may exist simultaneously in any medium. As long as the disturbances are linear, we may represent their total effect as the sum of the individual disturbances. But if the phenomenon is nonlinear, the total effect may depend on the cross product of the individual disturbances.

The engineer has a practical definition of nonlinear phenomena. As long as a simple filter can separate the different superposed periodic disturbances passing through a circuit, they are linear. But when the two or more disturbances have interacted in such a way that they cannot be so separated, then the phenomenon is nonlinear. Under certain circumstances where he wishes to induce artificial mixing, the engineer will use a nonlinear device such as a rectifier or detector.

Many of the differential equations of mathematical physics are linear in the first-order. They may contain, however, certain nonlinear terms that are negligible unless the variable is exceptionally large. 
Consider the following equation:

$$
\frac{d^{2} E}{d t^{2}}+\omega^{2} E+\beta E^{2}=0,
$$

where $\beta$ is small. When $\beta$ is zero, this equation has the solution:

$$
E=A \sin \omega t .
$$

But when we substitute this into the original equation, the term in $E$ becomes

$$
\beta E^{2}=\beta A^{2} \sin ^{2} \omega t=\frac{\beta A^{2}}{2}(1-\cos 2 \omega t) .
$$

In other words, the perturbations have caused the harmonic of frequency $2 \omega$ to appear.

To obtain a general solution, we may employ the complex Fourier series:

$$
E=\sum_{n=-\infty}^{\infty} A_{n} e^{i \omega n t}
$$

Substituting this into the differential equation, we get

$$
\sum A_{n} \omega^{2}\left(n^{2}-1\right) e^{i \omega n t}+\beta \sum \sum A_{m} A_{m}, e^{i \omega\left(m+m^{\prime}\right) t}=0 .
$$

Carry out the double summation by first summing over pairs of $m$ and $m^{\prime}$ such that

$$
m+m^{\prime}=n .
$$

Then we can write

$$
\sum_{n}\left[A_{n} \omega^{2}\left(n^{2}-1\right)+\beta \sum_{m} A_{m} A_{n-m}\right] e^{i \omega n t}=0 .
$$

Hence we must have

$$
A_{n} \omega^{2}\left(n^{2}-1\right)+\beta \sum_{m} A_{m} A_{n-m}=0 .
$$

This infinite set of simultaneous equations is, of course, nonlinear in the $A_{n}$ 's. The solution contains higher harmonics, though they may have progressively smaller amplitudes.

The most familiar nonlinear ionospheric process has received the name Luxembourg Effect, because it first showed up in connection with radio Luxembourg. The Luxembourg Effect is a particular example of a general phenomenon that might be more accurately termed "The Interaction of Acoustic and Electromagnetic Waves in an Ionized Medium." The medium may or may not contain a magnetic field.

In the Luxembourg Effect, a radio transmitter of high power produces an intense electromagnetic field in the ionosphere. If an audio frequency modulates the carrier, the electron velocity field responds to this modulation. In effect, the temperature of the electron gas fluctuates by an appreciable amount, according to the impressed audio frequency. This temperature variation may also be described as a pressure variation, a irue acoustic wave. I shall refer to the initial modulated wave and the acoustic disturbance it produces in the medium as the "unwanted wave."

The various propagation constants that depend on temperature or pressure of the electron gas in the medium vary with the frequency of the acoustic disturbance. If the density of the gas is high enough to make the medium dissipative, the imaginary part of the complex index of refraction also varies. A second radio wave, termed the "wanted wave," traversing the medium, will suffer from this variable absorption. Hence the wanted wave acquires some of the modulation of the unwanted wave. Since this superposed modulation constitutes an interference with the wanted wave, it is undesired or "unwanted." Thus the terminology.

The theory of this wave interaction was first given by Bailey and Martyn [1934], who pointed out the importance of magnetic fields, especially for radio waves near the gyrofrequency. The formulas they derived, however, were oversimplified, in that they linearized the equations describing the interaction. As a result, their formulas do not properly display the phase lag of the acoustic disturbance relative to the modulation by an amount dependent on the audio frequency. Menzel and Layzer have developed a more detailed theory which will be presented at this conference.

Experiments have shown that the Luxembourg variety of wave interaction rarely leads to cross modulation in excess of a few percent. The amount is very sensitive to a number of parameters. In the medium itself the significant variables are: the electron density and temperature, the frequency of dissipative electron collisions, and the magnetic field that fixes the electron gyrofrequency. The phenomenon further depends on the power and polarization, in addition to the radio and audio frequencies of the unwanted wave. The frequency and polarization of the wanted wave are also significant. The angle of incidence on the ionosphere determines the degree of penetration. Since the unwanted acoustic disturbance is localized, the greatest interaction will occur when the wanted waves travel through a significant portion of the disturbed volume.

Although the original theory and observations refer to the interaction of two separate and independent waves, examination of the basic equation shows that a single modulated wave in the medium may interact with itself. As before, the fluctuating field causes an acoustic disturbance in the medium. This results in a variable attenuation coefficient, which reacts on the original wave. Since the phase lag is a function of the acoustic frequency, the degree of attenuation will similarly vary with the acoustic frequency. In effect, some frequencies will experience demodulation whereas others will be excessively modulated.

The foregoing phenomenon, termed "self interaction," indicates that any acoustic disturbanceof whatever origin - can impress its modulation on 
a wave traversing the volume. Rockets, missiles, explosions, collisions of solar ion clouds with the upper atmosphere, etc., represent potential sources of such acoustic waves. The question requiring study is the magnitude of the interaction, not whether such interaction occurs. More specifically, of the various kinds of interaction, are there any that could be studied experimentally?

Certain difficulties are obviously present, resulting from fundamental differences in acoustic fields of radio origin on one hand and of mechanical origin on the other. For example, since the velocity of propagation of a radio wave greatly exceeds that of sound, the acoustic field induced by a modulated electromagnetic wave will be in phase over a large volume of space. Hence the interaction with the wanted wave will likewise be in phase over a large volume of space.

A mechanical disturbance, on the contrary, propagates with a much smaller velocity. Hence a wanted wave, progressing through the perturbed medium, will suffer high attenuation in certain zones and low attenuation in others. The effect resembles that of interference on optical frequencies. The problem is complex and the total effect on the wanted wave depends on the character and the extent of the basic disturbance.

The details of the interaction require precise analysis. The limited volume of the mechanical disturbance may well be offset by the intensity of the disturbance itself. In a region where shock waves may exist, numerous nonlinear effects may occur, complicating the transport of electromagnetic waves through the medium. The complex (dissipative) index of refraction will probably contain significant velocity-dependent terms of nonlinear character. The effect on a radio wave transversing the medium may still be appreciable. Magnetohydrodynamic compression of the gas will significantly alter the magnetic field, with attendant complication of the mathematical problem.

M. Cutolo has demonstrated experimentally the existence of a phenomenon sufficiently different from the Luxembourg Effect to deserve a special designation. He refers to it as the "detection effect," since it depends on nonlinear ionospheric properties for its occurrence. I prefer to call it the "Cutolo Effect," after its discoverer. I shall describe it briefly, since Cutolo plans to devote his paper to more conventional nonlinear problems.

Cutolo directs a beam of pulsed, modulated VHF waves upon the ionosphere. Of significance is his use of the gyro frequency as the modulation frequency. In the Luxembourg terminology, this pulsed, modulated, VHF wave, further modulated at audio frequencies to carry intelligence, represents the "unwanted wave." Cutolo reports that a second $\mathrm{HF}$ wave, the wanted wave, reflected from the ionospheric region traversed by the intense, focused VHF signal, will have the audio signal transferred to it. The amount of this cross modulation is very sensitive to the exactness of match between the gyro and modulating frequencies. Cutolo has found that the effect occurs for both vertical and oblique incidence of the wanted wave.

I have not seen, up to this time, any quantitative physical explanation of the Cutolo effect. An unwanted wave on the gyrofrequency would produce a large interaction of the Luxembourg type. But a $\mathrm{VHF}$ wave, modulated at the gyrofrequency, is not equivalent to a pure electromagnetic wave on the gyrofrequency. As is well known, such a wave can be expressed as an unmodulated carrier and two unmodulated sidebands separated from the carrier by the gyrofrequency. To account for the Cutolo effect we must suppose that some unknown ionospheric nonlinearity so mixes the carrier and sidebands as to release the true gyrofrequency. It is difficult to see, though, how such interactions occur because of the very high frequencies employed. Cutolo used a carrier of the order of $50 \mathrm{Mc} / \mathrm{s}$.

The following analysis will serve as an elementary introduction to ionospheric theory for younger scientists. The paper also considers various nonlinear effects. An electron of mass $m_{i}$ and charge $-\epsilon_{i}$, moving with vector velocity $\mathbf{v}_{i}$ in an electromagnetic field $\mathbf{E}$ and $\mathbf{H}$ and external acceleration $\mathbf{F}$, obeys the well-known equation of motion:

$$
m_{i} \frac{d \mathbf{v}_{i}}{d t}=-\epsilon_{i} \mathbf{E}-\frac{\epsilon_{i}}{c} \mathbf{v}_{i} \mathbf{x H}+m_{i} \mathbf{F}
$$

This equation applies along with Maxwell's equations to define the physical state of the medium and its interaction with the field.

The field $\mathbf{F}$ represents several varieties of mechanical actions. In a macroscopic problem involving the entire atmosphere, $\mathbf{F}$ will include the gravitational acceleration $g$. It also represents the collisional effects of nearby molecules, which may act as a sort of resistance proportional to the velocity and collision frequency $\nu$. As we shall note later on, this collisional term may be anisotropic, because of pressure gradients in the gas.

Maxwell's equations are:

$$
\begin{aligned}
& \operatorname{curl} \mathbf{H}=\boldsymbol{\nabla} \mathbf{x} \mathbf{H}=\frac{\kappa}{c} \frac{\partial \mathbf{E}}{\partial t}+\frac{4 \pi}{c} \mathbf{J}, \\
& \operatorname{curl} \mathbf{E}=\boldsymbol{\nabla} \mathbf{x} \mathbf{E}=-\frac{\mu}{c} \frac{\partial \mathbf{H}}{\partial t}
\end{aligned}
$$

$$
\operatorname{div} \mathbf{H}=\boldsymbol{\nabla} \cdot \mathbf{H}=0
$$

$$
\operatorname{div} \mathbf{E}=\boldsymbol{\nabla} \cdot \mathbf{E}=4 \pi \rho_{\epsilon} / \kappa,
$$

wherein $\mathbf{J}$ is the electric current, $\rho_{\epsilon}$ the density of electric charge, $\kappa$ the dielectric constant and $\mu$ the magnetic permeability. Simultaneous solution of 
these equations leads to

$$
\begin{gathered}
\nabla^{2} \mathbf{E}=\frac{\mu \kappa}{c^{2}} \frac{\partial^{2} \mathbf{E}}{\partial t^{2}}+\nabla \nabla \cdot \mathbf{E}, \\
\nabla^{2} \mathbf{H}=\frac{\mu \kappa}{c^{2}} \frac{\partial^{2} \mathbf{H}}{\partial t^{2}} .
\end{gathered}
$$

When $\rho_{\epsilon}$ is constant, the last term of (6) vanishes and we have the ordinary wave equations. For a plane wave, polarized in the $z x$ plane and traveling along the $z$-axis, we have

$$
\mathbf{E}=\mathbf{i} E_{0} e^{ \pm i \omega(t-n z / c)}
$$

where $\omega$ is the circular frequency and $c / n$ the velocity of light in the medium. Thus $n$ is the index of refraction. The quantity $n$ may be complex. The unit vectors $\mathbf{i}, \mathbf{j}$, $\mathbf{k}$ are the customary Cartesian set. This equation satisfies (6) when

$$
n^{2}=\mu \kappa .
$$

For nonmagnetic media, $\mu=1$. Hence $\kappa=1$ for free space. Part of our problem involves the determination of the effective $\kappa$ when the space contains electric currents and charges.

Return now to (1) in the form:

$$
m_{i} \frac{d \mathbf{v}_{i}}{d t}=-\epsilon_{i} \mathbf{E}-\frac{\epsilon_{i}}{c} \mathbf{v} \mathbf{x} \mathbf{H}-m_{i} \nu \mathbf{v}_{i} .
$$

The first term on the right represents electric forces and the second, magnetic forces. The third term, somewhat schematically, represents the resistance that the charged particle encounters as it moves through the medium. The quantity $\nu$ is the number of collisions per second experienced by the moving ion. For convenience we shall drop the subscript $i$. The electrons, because of their great mobility, are the major contributors to the current density $\mathbf{J}$.

Thus:

$$
\mathbf{J}=-N \epsilon \mathbf{v}
$$

where $N$ is the number of electrons per $\mathrm{cm}^{3}$. Under different assumptions we shall calculate the variations of $\mathbf{v}$ and $\mathbf{J}$ with the time. We shall then substitute the $\mathbf{J}$, so calculated, into Maxwell's equation (2).

Case I. $\mathbf{H}=0, \nu=0, \mathbf{E}=$ const: $\mathbf{v}=-\epsilon E t / m$.

Case II. $\quad \mathbf{E}=0, \nu=0, \mathbf{H}=$ const.

Here take the derivative of (10) with respect to the time. The resulting equation reduces as follows:

$$
\frac{d^{2} \mathbf{v}}{d t}=-\frac{\epsilon}{m c}\left(\frac{d \mathbf{v}}{d t} \mathbf{x H}\right)=\left(\frac{\epsilon}{m c}\right)^{2}(\mathbf{v} \mathbf{x H}) \mathbf{x H} .
$$

Let $\mathbf{v}$ have two components $\mathbf{v}_{p}$ parallel to and $\mathbf{v}_{s}$ perpendicular (German: senkrecht) to the field. The triple vector product reduces to:

so that:

$$
(\mathbf{v} \mathbf{x H}) \mathbf{x H}=H^{2} \mathbf{v}_{s}
$$

and

$$
\frac{d^{2} \mathbf{v}_{s}}{d t^{2}}=\omega_{L}^{2} \mathbf{v}_{s} ; \frac{d^{2} \mathbf{v}_{p}}{d t^{2}}=0,
$$

$$
\mathbf{v}_{s}=\mathbf{v}_{0 s} e^{i \omega_{L} t} ; \mathbf{v}_{p}=\mathbf{v}_{0 p} ; \omega_{L}=\epsilon H / m c .
$$

The particle executes a spiral path with the Larmor frequency $\omega_{L}$. The radius, $a$, and pitch, $p$, of the spiral are

$$
a=v_{0 s} / \omega_{L}, p=v_{0 p} / \omega_{L} .
$$

Case III. $\mathbf{E}=0, \mathbf{H}=0, \nu=$ const; $\mathbf{v}=\mathbf{v}_{0} e^{-\nu t}$,

corresponding to simple damping.

Case IV. $E=E_{0} e^{i \omega t}, \mathbf{H}=0, \nu=0$,

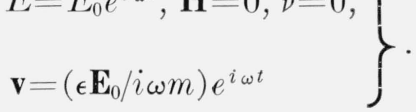

Case V. At this point, let us consider a more general problem for which we assume an electromagnetic wave traveling parallel to the $z$-axis. Let $E_{x}$ and $E_{y}$ be the vector electric fields and $H_{x}$ and $H_{y}$ the variable magnetic fields associated with the radiation. Let the magnetic field of the ionosphere be of magnitude $H$, in the direction of the propagation.

The general equation of motion, (10), breaks up into equations for the three components:

$$
\left.\begin{array}{l}
\frac{d v_{x}}{d t}=-\frac{\epsilon}{m} E_{x}-\frac{\epsilon H}{m c} v_{y}-\nu v_{x} \\
\frac{d v_{y}}{d t}=-\frac{\epsilon}{m} E_{y}+\frac{\epsilon H}{m c} v_{x}-\nu v_{y} \\
\frac{d v_{z}}{d t}=-\nu v_{z}
\end{array}\right\} .
$$

Multiply the second equation by $i$ and add to the first, employing (16). 'The result is

$$
\frac{d}{d t}\left(v_{x}+i v_{y}\right)=\left(i \omega_{L}-\nu\right)\left(v_{x}+i v_{y}\right)-\frac{\epsilon}{m}\left(E_{x}+i E_{y}\right) .
$$

Maxwell's field equations become:

$$
\left.\begin{array}{l}
\frac{\partial E_{y}}{\partial z}=\frac{1}{c} \frac{\partial H_{x}}{\partial t}, \frac{\partial E_{x}}{\partial z}=-\frac{1}{c} \frac{\partial H_{y}}{\partial t}, \\
-\frac{\partial H_{y}}{\partial z}=\frac{\kappa}{c} \frac{\partial E_{x}}{\partial t}-\frac{4 \pi N \epsilon v_{x}}{c}, \\
\frac{\partial H_{x}}{\partial z}=\frac{\kappa}{c} \frac{\partial E_{y}}{\partial t}-\frac{4 \pi N \epsilon v_{y}}{c}
\end{array}\right\} .
$$


Multiply the second and fourth of these equations by $i$ and combine as before, to give:

$$
\begin{aligned}
\frac{\partial}{\partial z}\left(E_{x}+i E_{y}\right) & =\frac{i}{c} \frac{\partial}{\partial t}\left(H_{x}+i H_{y}\right) \\
\frac{\partial}{\partial z}\left(H_{x}+i H_{y}\right) & =-\frac{\kappa i}{c} \frac{\partial}{\partial t}\left(E_{x}+i E_{y}\right)+\frac{4 \pi i N \epsilon}{c}\left(v_{x}+i v_{y}\right) .
\end{aligned}
$$

Now introduce the complex quantities:

$$
\left.\begin{array}{l}
v_{x}+i v_{y}=V e^{ \pm i \omega(t-n z / c)} \\
E_{x}+i E_{y}=E e^{ \pm i \omega(t-n z / c)} \\
H_{x}+i H_{y}=H e^{ \pm i \omega(t-n z / c)}
\end{array}\right\}
$$

where $V, E$, and $H$ are complex constants. With the aid of (24), (21) and (23) become:

$$
\begin{gathered}
{\left[i\left(-\omega_{L} \pm \omega\right)+\nu\right] V=-\epsilon E / m} \\
i n E=H \\
i \omega n H=-\kappa \omega E \pm 4 \pi i N \epsilon V .
\end{gathered}
$$

These equations contain four complex unknowns: $E, H, V$, and $n$. Of these, $n$ is the only one uniquely determined. $H$ and $V$ are, as one would expect, merely proportional to $E$. We may solve directly for $n^{2}$ :

$$
n^{2}=1-\frac{4 \pi N \epsilon^{2}}{m \omega} \frac{1}{\omega \mp} \frac{1}{\omega_{L} \mp i \nu},
$$

the complex index of refraction. Let

$$
r_{v}=r_{u_{r}} \mp i k,
$$

where $n_{r}$ and $n_{k}$ are real. Then:

$$
\begin{gathered}
n_{\tau}^{2}-k^{2}=1-\frac{4 \pi N \epsilon^{2}}{m \omega} \frac{\omega \mp \omega_{L}}{\left(\omega \mp \omega_{L}\right)^{2}+\nu^{2}}, \\
2 r_{r} k=\frac{4 \pi N \epsilon^{2}}{m \omega} \frac{\nu}{\left(\omega \mp \omega_{L}\right)^{2}+\nu^{2}} .
\end{gathered}
$$

The propagation factor becomes:

$$
e^{ \pm i \omega(t-n z / c)}=e^{ \pm i \omega\left(t-n_{r} z / c\right)-\omega k z / c} .
$$

The last term of the exponent indicates an amplitude that decreases exponentially with the distance. The effect results from the collisions, which transfer a certain amount of energy to the medium.

At this point, note the basic philosophy behind these elementary derivations of the propagation parameters. We have solved the general equation of motion to determine the velocity of the electron under the influence of external fields of force, electromagnetic, magnetic, and collisional. Our first objective was the calculation of the current density, $J$. Then, substituting this back into Maxwell's equation (2), we derived, in effect the dielectric constant $\kappa$, which by (9) is equal to the square of the complex index of refraction. Equation (2) thus takes the successive forms:

$$
\operatorname{curl} \mathbf{H}=\frac{1}{c} \frac{\partial \mathbf{E}}{\partial t}+\frac{4 \pi \mathbf{J}}{c}=\frac{1}{c} \frac{\partial \mathbf{E}}{\partial t}-\frac{4 \pi N \epsilon \mathbf{V}}{c}=\frac{\kappa}{c} \frac{\partial \mathbf{E}}{\partial t}=\frac{i \omega \kappa \mathbf{E}}{c},
$$

since $\mathbf{v}, \mathbf{E}$, and their derivations all have the same time dependence, $e^{i \omega t}$.

The physical significance of $\kappa$ becomes more meaningful, perhaps, if we follow the steps indicated by (33). Here we may use the complex form of (2),

$$
\begin{aligned}
\operatorname{curl} \mathbf{H} & =\frac{1}{c} \frac{\partial \mathbf{E}}{\partial t}+\frac{4 \pi \mathbf{J}}{c} \\
& =\left( \pm \frac{i \omega E}{c}-\frac{4 \pi N \epsilon V}{c}\right) e^{ \pm i \omega(t-n z / c)} \\
& = \pm i \omega+4 \pi N \epsilon^{2} \\
& =\left[ \pm \frac{i \omega}{c}+\frac{4 \pi N \epsilon^{2}}{m c} \frac{1}{i\left(-\omega_{L} \pm \omega\right)+\nu}\right] E e^{ \pm i \omega(t-n z / c)} \\
& = \pm \frac{i \omega \kappa}{c} E e^{ \pm i \omega(t-n z / c)}
\end{aligned}
$$

where we have used (25) to express $V$ in terms of $E$. Now, solving for $\kappa$ we get

$$
\kappa=n^{2}=1-\frac{4 \pi N \epsilon^{2}}{\omega} \frac{1}{\omega \mp \omega_{L} \mp i \nu}
$$

as before.

Now note some of the assumptions implicit in these derivations of $\kappa$. In the strictest sense we should have a different velocity, $\mathbf{v}_{i}$, for each electron. We would then have evaluated the average $\mathbf{v}$ and $\mathbf{J}$ as follows:

$$
\mathbf{J}=-\epsilon N \mathbf{v}=-\epsilon \sum \mathbf{v}_{i}=-\epsilon \int \mathbf{v} f(v) d v
$$

where $f(v)$ is the velocity distribution function for the electron gas.

The solution of this problem is far more difficult than appears at first sight. The function $f=f(v)$, will generally depend on time and position. The Boltzmann transport equation is basic for the determination of $f$.

$$
\frac{d f}{d t}=\frac{\partial f}{\partial t}+\mathbf{v} \cdot \boldsymbol{\nabla} f+\boldsymbol{\alpha} \cdot \boldsymbol{\nabla}_{v} f+S=0 .
$$

In the above, $\alpha$ is the acceleration and $\boldsymbol{\nabla}_{v}$ the gradient in velocity space. The quantity, $S$, is the collision integral, which depends on the rate of change in the distribution function resulting from collisions. This 
term takes account of the creation or destruction of electrons in a given range of velocity. To calculate $S$ we must know the physical details of both elastic and inelastic collisions.

The first three terms of (37) are vector abbreviations for the purely mathematical concept of the total derivative of a function, $f$, that depends on the time, on the coordinates, and the velocity components. In rectangular coordinates, we may write

$$
\begin{array}{r}
\frac{d}{d t} f(x, y, z, u, v, \omega, t)=\frac{\partial f}{\partial t}+\frac{\partial f}{\partial x} \frac{d x}{d t}+\frac{\partial f}{\partial y} \frac{d y}{d t}+\frac{\partial f}{\partial z} \frac{d z}{d t} \\
+\frac{\partial f}{\partial u} \frac{d u}{d t}+\frac{\partial f}{\partial v} \frac{d v}{d t}+\frac{\partial f}{\partial \omega} \frac{d \omega}{d t}+S=0
\end{array}
$$

wherein $u, v$, and $\omega$ are the three velocity components.

Let $\mathbf{v}^{\prime}$ and $\mathbf{v}$ be the vector velocities of the electron prior to and after the collision. Let $\mathbf{v}_{1}^{\prime}$ and $\mathbf{v}_{1}$ be the initial and final velocities of the colliding particle. Let $f\left(\mathbf{v}^{\prime}\right) d \mathbf{v}^{\prime}$ and $f(\mathbf{v}) d \mathbf{v}$ symbolically represent the number of electrons within the ranges $d \mathbf{v}^{\prime}$ and $d \mathbf{v}$, and let $F\left(\mathbf{v}_{1}^{\prime}\right) d \mathbf{v}_{1}^{\prime}$ and $F\left(\mathbf{v}_{1}\right) d \mathbf{v}_{1}$ similarly represent the number of colliding particles in ranges $d \mathbf{v}_{1}^{\prime}$ and $d \mathbf{v}_{1}$.

The relative velocity of collision becomes:

$$
\mathbf{u}=\mathbf{v}-\mathbf{v}_{1} \text { and } u=\left|\mathbf{v}-\mathbf{v}_{1}\right| \text {. }
$$

Let $\sigma(u, \theta)$ be the differential scattering cross section describing the probability that a colliding electron will be deflected through an angle $\theta$, during collision. Define the solid angle $d \Omega=\sin \theta d \theta d \phi$. Then we may express $S$ as follows:

$$
S=\iint d \mathbf{v}_{1} d \Omega \sigma(u, \theta) u\left[f(\mathbf{v}) F\left(\mathbf{v}_{1}\right)-f\left(\mathbf{v}^{\prime}\right) F\left(\mathbf{v}_{1}^{\prime}\right)\right] .
$$

The problem of calculating $S$ and $f$ depends on a precise knowledge of the target cross section, $\sigma$, as a function of $u$ and $\theta$. We shall not consider it further here, except to note that our arbitrary representation of the collisional effects by the term $m \nu \mathbf{v}$ in (10) was naive, to say the least. It was particularly an oversimplification in that we assumed $\nu$ to be independent of $\mathbf{v}$, in order to obtain a linear equation.

We should have been much more realistic if we had assumed $\nu$ varied with the velocity. Certainly the collision frequency will depend on the temperature of the electron gas. This temperature, in turn, will vary with the impressed electric field. And if the field is modulated, the modulation frequency will cause $\nu$ to vary at that frequency. Thus the absorption coefficient, $k$, (31), should also vary with the time. It is this variable absorption that leads to cross modulation, the Luxembourg effect, gyrointeraction and other nonlinear responses, some of which we shall be discussing during this conference.

But additional, still less obvious assumptions exist in our oversimplified theoretical discussion. Maxwell's equations apply, of course. We have ignored possible reactions of the ionospheric plasma with the terrestrial magnetic field. I refer particularly to phenomena lying in the realm of magnetohydrodynamics. We can at least infer the character of some of these problems.

Rewrite (1) in the general form:

$$
m_{i} \frac{d \mathbf{v}_{i}}{d t}=\epsilon_{i} \mathbf{E}-\frac{\boldsymbol{\epsilon}_{i} \mathbf{v}_{i} \mathbf{x} \mathbf{H}}{c}+m_{i} \mathbf{F},
$$

wherein a positive $\epsilon_{i}$ refers to an ion and a negative $\epsilon_{i}$ to an electron. We can take averages over a unit volume as follows:

$$
\begin{aligned}
\rho & =\sum m_{i} \\
\rho \mathbf{v} & =\sum m_{i} \mathbf{v}_{i} \\
\mathbf{J} & =\sum \epsilon_{i} \mathbf{v}_{i} \\
\rho_{\epsilon} & =\sum \epsilon_{i}
\end{aligned}
$$

wherein $\rho$ is the mass per unit volume. These summations imply, when relevant, integration over the velocity-distribution functions previously discussed.

The force-field $\mathbf{F}$ breaks up into two parts; external fields such as gravity and internal fields which result from colliding particles. One may write the latter force on a given particle, $i$, as follows:

$$
\mathbf{F} \text { int }=\sum_{j} \mathbf{F}_{i j}
$$

where the summation is taken over all neighboring particles $j$. This term cancels out only when the medium is uniform. If electric currents are present, the term gives rise to forces simulated in our earlier treatment by the collisional term, $m_{i} \nu \mathbf{v}$. This force can also be expressed, for the entire medium, as a sort of electrical resistance, $R$, in the form $R \mathbf{J}$. It also produces such major forces as pressure gradients. Tangential forces, in the presence of sheer, lead to the phenomenon of viscosity. In the presence of temperature gradients it can even cause thermoelectric effect. For example we may write

$$
\mathbf{E}=R(\mathbf{J}-\theta \nabla T),
$$

where $\theta$ is a thermoelectric coefficient. In the strictest sense both $R$ and $\theta$ will be tensors rather than scalars.

As we sum (41) over the individual particles and employ Maxwell's equations we obtain the general equation of magnetohydrodynamic motion:

$$
\begin{aligned}
& \rho \frac{d \mathbf{v}}{d t}=-\boldsymbol{\nabla} p+\rho \mathbf{F}_{\mathrm{exp}}+\frac{1}{4 \pi}(\boldsymbol{\nabla} \times \mathbf{H}) \times \mathbf{H}+\frac{1}{4 \pi}(\boldsymbol{\nabla} \times \mathbf{E}) \\
& \times \mathbf{E}+\frac{1}{4 \pi} \mathbf{E} \nabla_{1} \cdot \mathbf{E}-\frac{1}{4 \pi c} \frac{\partial}{\partial t}(\mathbf{E} \times \mathbf{H}) .
\end{aligned}
$$


When $H$ and $E$ are constant or zero, the equatio reduces to that of ordinary hydrodynamics, less the second-order terms related to viscosity. The terms represent the forces arising from the electromagnetic fields. $\mathbf{E} \times \mathbf{H}$ is the Poynting vector, which represents energy flowing out of the volume element. Hence the partial time derivative of $\mathbf{E} \times \mathbf{H}$ represents the forces caused by radiation pressure.

Now multiply (31) by $\epsilon / m$ and sum again. The result is

$$
\frac{m}{n \epsilon^{2}} \frac{d \mathbf{J}}{d t}=\mathbf{E}+\frac{1}{c}(\mathbf{v} \times \mathbf{H})-R(\mathbf{J}-\theta \nabla T)
$$

where $m$ is the mass of an electron and $n$ the number of electrons per unit volume. In taking this sum we have allowed for the fact that electrons, by virtue of their small mass, are the main contributors to the electric currents. In passing we may note that this equation has several obvious limiting forms. It can reduce to Ohms law

$$
\mathbf{E}=R \mathbf{J},
$$

or to the electromotive force induced in a conductor moving through a magnetic field

$$
\mathbf{E}=-(\mathbf{v} \times \mathbf{H}) / c .
$$

Even (46) has its limitations. Acoustic waves or hydrodynamic waves can directly produce changes in $\mathbf{J}$, by simple compression or expansion of the current-bearing regions. Moreover, in a conducting medium, $\mathbf{H}$ itself may change under magnetohydrodynamic forces. Such changes in $\mathbf{H}$ produce fluctuations in $\mathbf{J}$. As we have just noted, variations in J directly affect the dielectric constant. We must, therefore, set up the equations governing the variations, not only of $\mathbf{H}$ but also of $\mathbf{E}$.

The following analysis is by no means complete. However, consider the steady-state situation, derived from (46):

$$
\mathbf{E}+(\mathbf{v} \times \mathbf{H}) / c-R \mathbf{J}=0 .
$$

Take the curl of this equation and reduce by means of the Maxwell relationships. We get:

$$
\begin{aligned}
\frac{d \mathbf{H}}{d t} & =\frac{\partial \mathbf{H}}{\partial t}+\mathbf{v} \cdot \boldsymbol{\nabla} \mathbf{H} \\
& =\mathbf{H} \cdot \boldsymbol{\nabla} \mathbf{v}-\mathbf{H} \boldsymbol{\nabla} \cdot \mathbf{v}+\frac{c^{2} R}{4 \pi}\left[\nabla^{2} H-\frac{\kappa}{c^{2}} \frac{\partial^{2} \mathbf{H}}{\partial t^{2}}\right]
\end{aligned}
$$

Now take the partial time derivative of (49) and derive a similar equation for $\mathbf{E}$. The result is:

$$
\frac{\partial \mathbf{E}}{\partial t}+\frac{1}{c} \frac{\partial}{\partial t}(\mathbf{v} \times \mathbf{H})=\frac{c^{2} R}{4 \pi}\left[\nabla^{2} \mathbf{E}-\frac{\kappa}{c^{2}} \frac{\partial^{2} \mathbf{E}}{\partial t^{2}}-\nabla \nabla \cdot \mathbf{E}\right] .
$$

These equations are also subject to the equation of continuity,

or

$$
\frac{d \rho}{d t}=\frac{\partial \rho}{\partial t}+\mathbf{v} \cdot \boldsymbol{\nabla} \rho=-\rho \boldsymbol{\nabla} \cdot \mathbf{v}
$$

$$
\frac{\partial \rho}{\partial t}+\nabla \cdot(\rho \mathbf{v})=0
$$

For regions where the conductivity is high, $R \rightarrow 0$ and we recover the ordinary equations of magnetohydrodynamics. When the conductivity is low, divide through by $R$. The electromagnetic equations then reduce to the simple wave equations for $\mathbf{E}$ and $\mathbf{H}$. In other words, for media of low conductivity, the hydrodynamic and electromagnetic fields are independent. Otherwise the two fields are coupled. This means that, in effect, we can combine the purely dynamic terms into a nonlinear wave equation, in which the dielectric constant is a function of the dynamical variations.

I shall not attempt to carry the analysis further. My primary objective has been to show that nonlinear effects abound in this general problem. I should point out one additional fact. The electrical resistance $R$ is closely related to the damping factor $k$. Since $k$, in turn, depends on both electrical and dynamical factors, the factor $R$ introduces additional nonlinearities. Detailed solutions depend on assumptions concerning the ba ic physical conditions in the medium. I have especially desired to emphasize the importance of dynamical or magnetohydrodynamical effects on radio propagation.

\section{References}

Bailey, V. A. (Jan. 9, 1937a), Interaction by resonance of radio waves, Nature $\mathbf{1 3 9}, 68-69$.

Bailey, V. A. (May 15, 1937b), Resonance in the interaction of radio waves, Nature 139, 838-839.

Bailey, V. A. (April 1937c), The motions of electrons in the presence of variable electric fields and a constant magnetic field, Phil. Mag. 23, No. 156, 774-791.

Bailey, V. A. (1956), On the interaction of radio waves, Nuovo Cimento 4, Suppl. 4, 1430-1449.

Bailey, V. A., and D. F. Martyn (Aug. 1934), The influence of electric waves on the ionosphere, Phil. Mag. 18, No. 118, 369-385.

Ginsberg, V. L., and A. V. Gurevich (1960), Nonlinear phenomena in a plasma located in a alternating electromagnetic field, Usp. Fiz. Nauk $\mathbf{7 0 ,} 201-246$ and $393-428$. English translation, Soviet Physics, Uspekhi 3, 115-146 and $175-284$.

(Paper 69D1-438) 